УДК 004

DOI $10.21661 / \mathrm{r}-497643$

Н.К. Петрова, Т.М. Алимов, М.Б. Алабдуллах, А.Х.Х. Альхузайи, Р.3. Каттави ДИНАМИЧЕСКИЕ МАССИВЫ НА С++ И ЗАДАЧА ПОИСКА МИНИМАКСОВ

Аннотация: в представленной статье рассматривается классическая задача поиска седловой точки в двумерном массиве. Подобного рода задачи весьма востребованы в геодезии, при построении топографических или гравиметрических карт местности, в энергетике - при планировании нагрузки на электрические или тепловые сети. Код программ предлагаемого проекта написан в рамках структурного подхода на языке C++ в среде разработки Visual Studio 2017.

Ключевые слова: седловая точка, VS Studio, C++, динамические массивы.

N.K. Petrova, T.M. Alimov, M.B. Alabdullah, A.H.H. Alhuzaji, R.Z. Kattavi

\title{
DYNAMIC ARRAYS IN C ++ AND MINIMAX SEARCH PROBLEM
}

Abstract: the article presents the classical saddle-point problem in a two-dimensional array. Such tasks are highly demanded in geodesy, in the construction of topographic or gravimetric maps of the terrain, in the energy sector - in planning the load on electric or thermal networks. The program code of the proposed project is written within the framework of the structured approach in the $C++$ language in the Visual Studio 2017 programming environment.

Keywords: saddle point, VS Studio, $C++$, dynamic arrays.

Введение. Возможности операций с динамическими массивами на $\mathrm{C}++$ позволяет без особых затруднений решать классические задачи на их основе для закрепления навыков выполнения низкоуровневых операций при работе с отдельными элементами массива, а также передаче массивов в другие функции через ссылки и указатели, строя проект в рамках технологии структурного программирования. Дадим определение 
ключевому понятию «седловая точка», рассматриваемого в нашей статье. Седловая точка - элемент матрицы, являющуюся наименышим в своей строке и одновременно наибольшим в своем столбце или, наоборот, является наибольшим в своей строке и наименышим в своем столбце. Составим программный проект, который для любого двумерного массива размера $(N \times M)$, где $\mathrm{N}$ - количество строк, $\mathrm{M}$ - количество столбцов, находит индексы седловых точек и их количество. Программу будем реализовывать в среде программирования Visual Studio 2017 на языке C++.

Контрольные примеры для разработки алгоритма и обеспечения полнотьл программы. Для реализации проекта необходимо начать разработку с составления контрольных примеров. В качестве контрольного примера для тестирования рассмотрим двумерный массив размером $(\mathrm{n}=3 ; \mathrm{m}=4)$, заполним его числами в пределах $[0 ; 100]$ (рис. 1).

\begin{tabular}{|r|r|r|r|r|}
\hline & 0 & 1 & 2 & 3 \\
\hline 0 & 45 & 84 & 25 & 17 \\
\hline 1 & 60 & 72 & 33 & 1 \\
\hline 2 & 80 & 97 & 47 & 29 \\
\hline
\end{tabular}

Рис. 1. Исходный массив

Далее найдем максимальные элементы в каждой строке (рис. 2):

\begin{tabular}{|r|r|r|r|r|}
\hline & 0 & 1 & 2 & 3 \\
\hline 0 & 45 & 84 & 25 & 17 \\
\hline 1 & 60 & 72 & 33 & 1 \\
\hline 2 & 80 & 97 & 47 & 29 \\
\hline
\end{tabular}

Рис. 2. Максимальные элементы строк

Далее найдем минимальные элементы в каждой строке (рис. 3):

\begin{tabular}{|r|r|r|r|r|}
\hline & 0 & 1 & 2 & 3 \\
\hline 0 & 45 & 84 & 25 & 17 \\
\hline 1 & 60 & 72 & 33 & 1 \\
\hline 2 & 80 & 97 & 47 & 29 \\
\hline
\end{tabular}

Рис. 3. Минимальные элементы строк

Далее найдем максимальные элементы в каждом столбце (рис. 4): 


\begin{tabular}{|r|r|r|r|r|}
\hline & 0 & 1 & 2 & 3 \\
\hline 0 & 45 & 84 & 25 & 17 \\
\hline 1 & 60 & 72 & 33 & 1 \\
\hline 2 & 80 & 97 & 47 & 29 \\
\hline
\end{tabular}

Рис. 4. Максимальные элементы столбцов

Далее найдем минимальные элементы в каждом столбце (рис. 5):

\begin{tabular}{|r|r|r|r|r|}
\hline & 0 & 1 & 2 & 3 \\
\hline 0 & 45 & 84 & 25 & 17 \\
\hline 1 & 60 & 72 & 33 & 1 \\
\hline 2 & 80 & 97 & 47 & 29 \\
\hline
\end{tabular}

Рис. 5. Минимальные элементы столбцов

Далее сравниваем максимальные элементы строк и минимальные элементы столбцов, и наоборот, минимальные элементы строк и максимальные элементы столбцов, если они совпадают, то записываем индексы этой точки и увеличиваем количество седловых точек на 1.

Проанализировав результаты, видим, что есть 2 седловые точки с индексами $(1 ; 1)$ и $(2 ; 3)$ (рис. 6):

\begin{tabular}{|r|r|r|r|r|}
\hline & 0 & 1 & 2 & 3 \\
\hline 0 & 45 & 84 & 25 & 17 \\
\hline 1 & 60 & 72 & 33 & 1 \\
\hline 2 & 80 & 97 & 47 & 29 \\
\hline
\end{tabular}

Рис. 6. Седловые точки

Анализ контрольных примеров показывает, что решение данной задачи требует использование следующих алгоритмических методов:

- введение счётчика. Переменная k для подсчета количества седловых точек;

- использование операторов ввода/вывода. Операторы cin и cout;

- использование циклических алгоритмов: for и while;

- работа с индексами двумерного массива. $N$ - количество строк, $M$ - количество столбцов массива $a$; 
- введение временных переменных. Min - минимальный элемент строки; Maxмаксимальный элемент строки; MinCol-минимальный элемент столбца; MaxCol-максимальный элемент столбца;

- нахождение максимума;

- нахождение минимума;

- передача массива в функции. Массив передаётся в функции через указатели на указатели. Функция MakeArr - создает массив, print - выводит массив, solution - вычисляет седловые точки массива и выводит их индексы на экран.

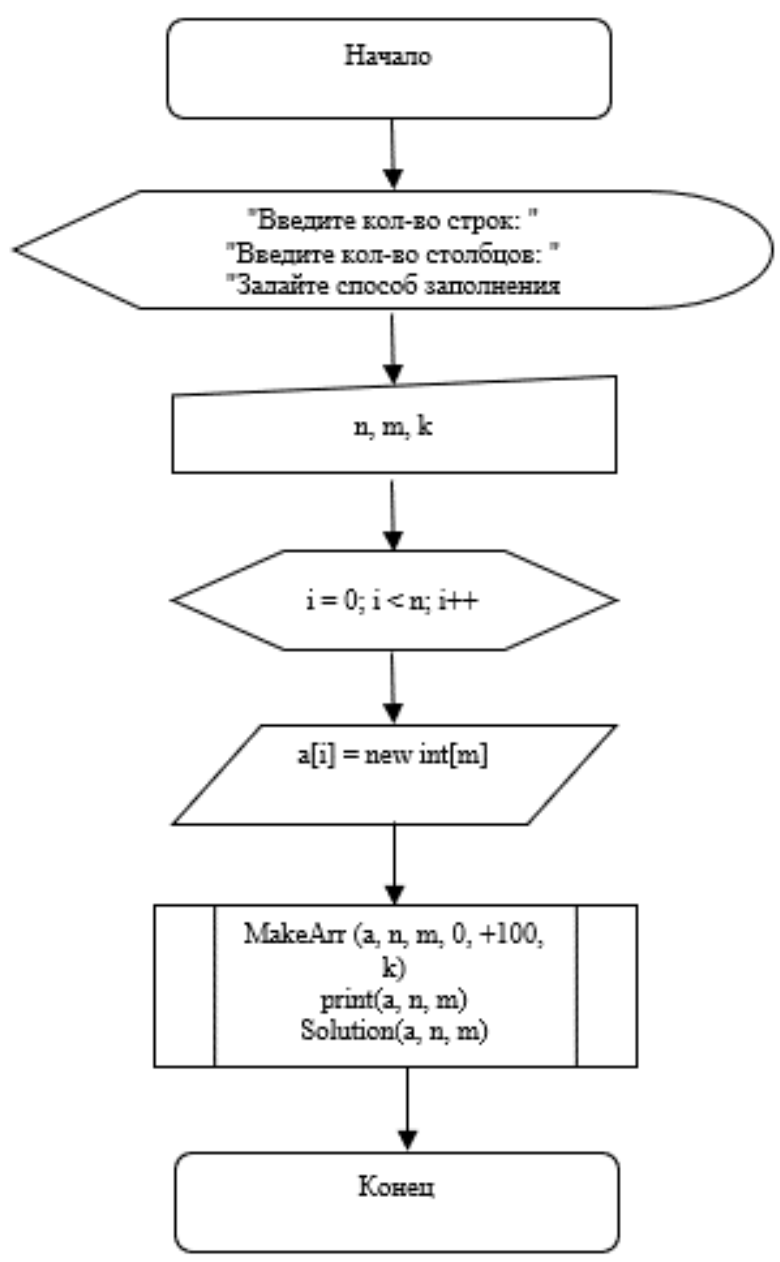

Рис. 7. Главная программа

В качестве примера приведем текст функции MakeArr:

void MakeArr(int **tf, int nf, int mf, int r_min, int r_max, int way)

srand((unsigned int)time(NULL)); 


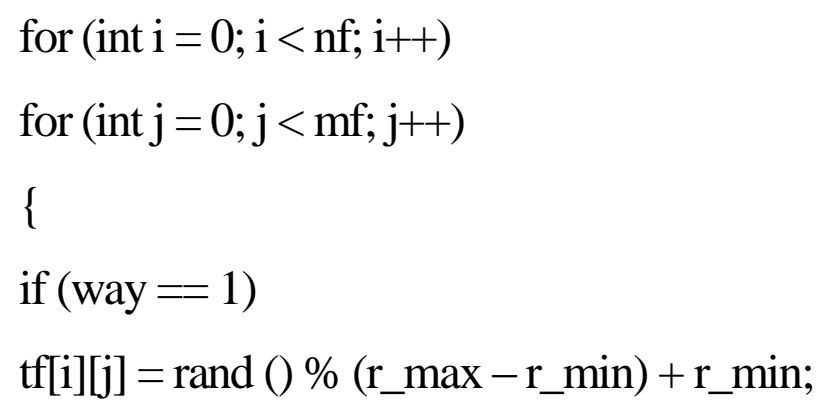

else

\{

cout $<<$ «Элемент (" << i <<"; " << j <<"): ";

$\operatorname{cin} \gg \mathrm{tf}[\mathrm{i}][\mathrm{j}]$;

\}

\}

return;

\}

Int tf - исходный массив; int $n f$ - количество строк; int $m f$ - количество столбцов; int r_min - левая граница генерируемых чисел; int $r \_m a x$ - правая граница генерируемых чисел; int way - флаг, введенный для выбора способа инициализации массива. Если он равен 1 , то инициализация идёт с помощью генератора случайных чисел в заданном диапазоне значений от $r \_$min до $r \_m a x$, в противном случае инициализация осуществляется вводом значений с консоли.
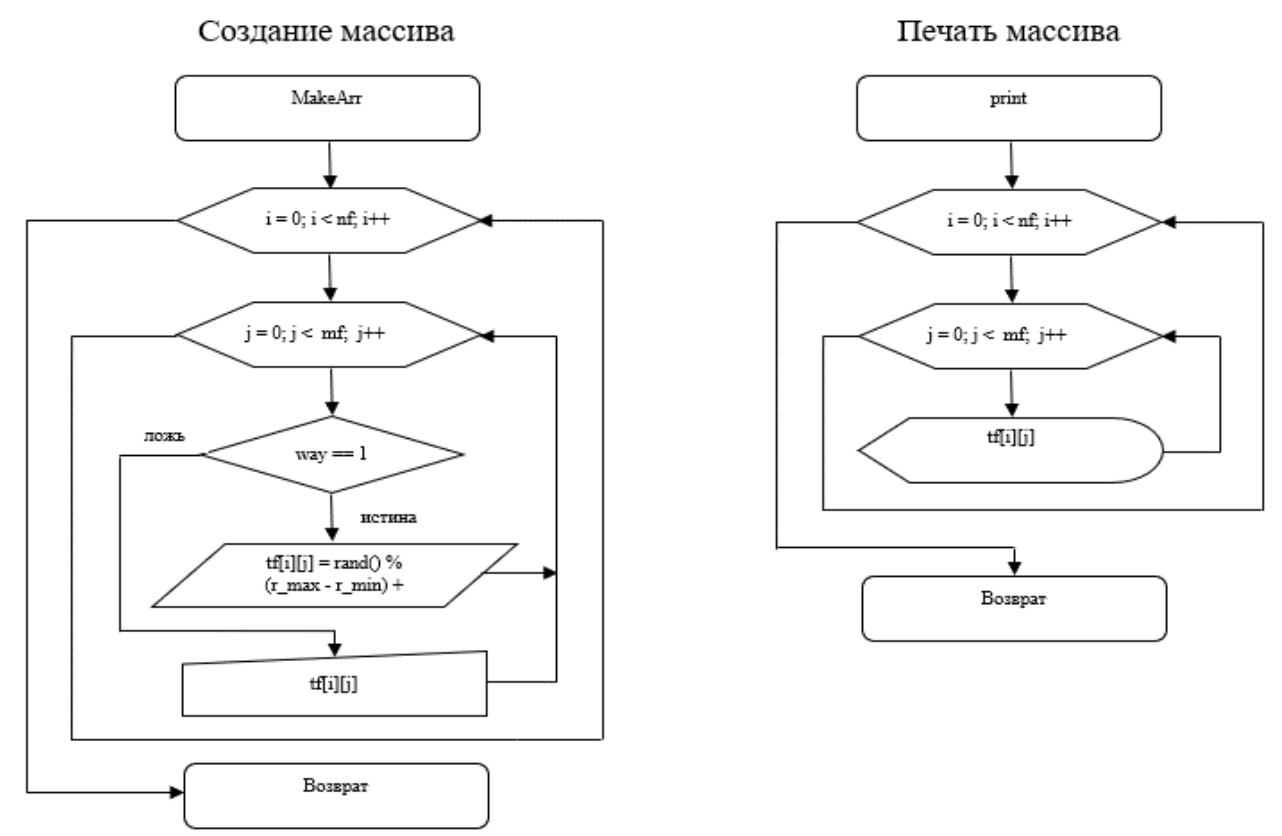

Рис. 8. Создание и печать массива 
Заключение. По итогам проведенной работы нами разработан алгоритм, использующий приемы структурного программирования, для нахождения седловых точек в двумерном массиве, визуализированный словесно-блочной схемой, разработан контрольный пример для проверки корректности программного кода. Программу протестировали 3 раза:

На 2 способа инициализации двумерного массива (1-2).

На неправильно введенный размер двумерного массива (3).

Результаты отладки программы в среде разработки Visual Studio 2017 представлены ниже:

1) способ инициализации вручную с консоли $k=0$;

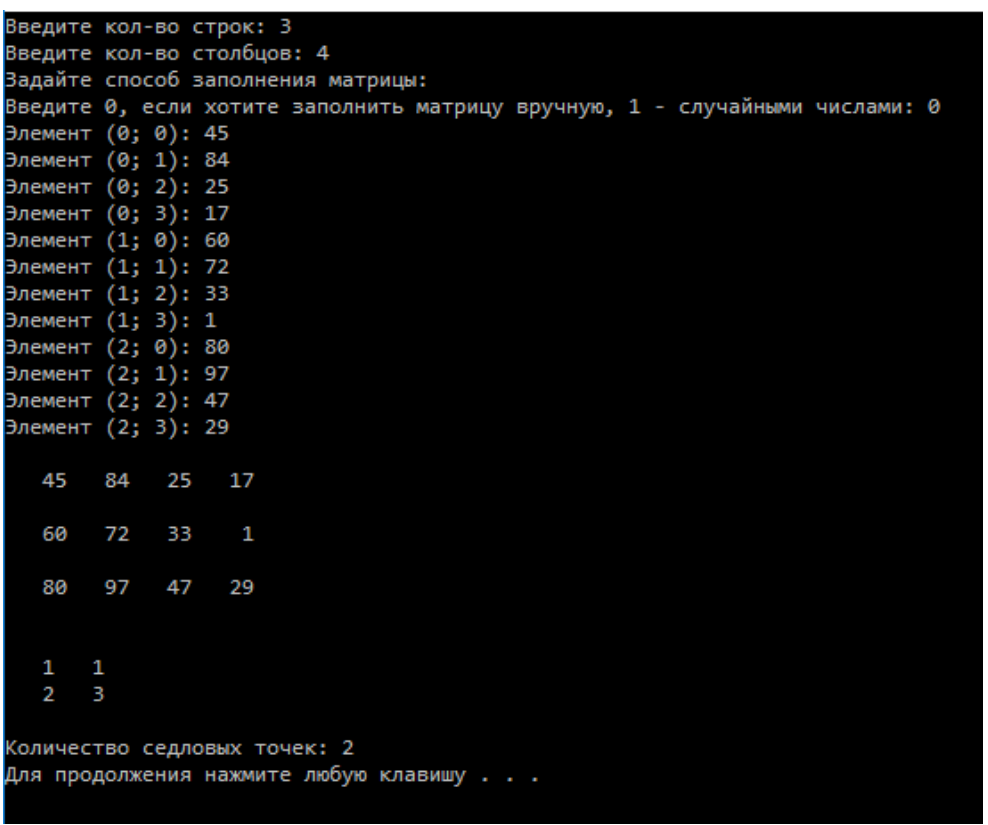

Рис. 7. Способ инициализации вручную с консоли

2) способ инициализации случайными числами $k=1$;

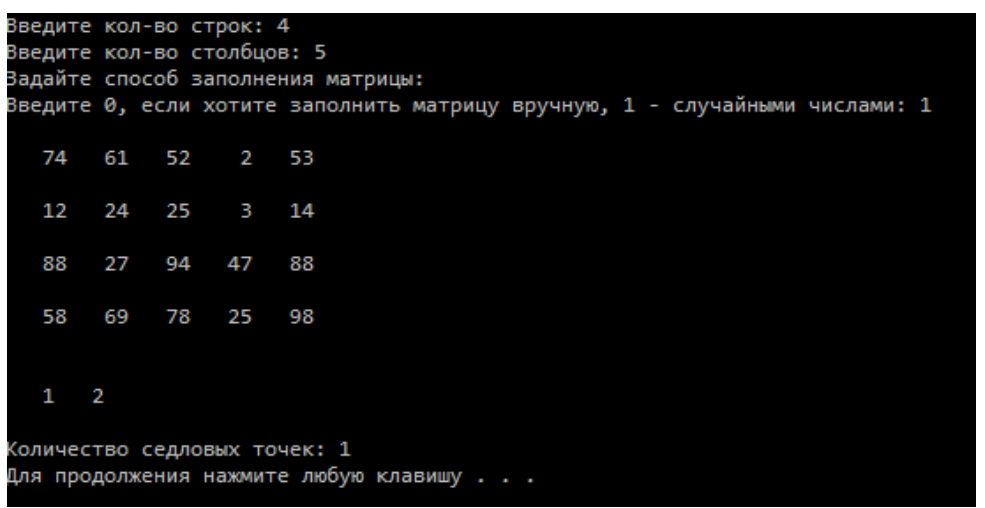

Рис. 8. Способ инициализации случайными числами 
3) неправильно введенный размер двумерного массива: $n=0, m<0$.

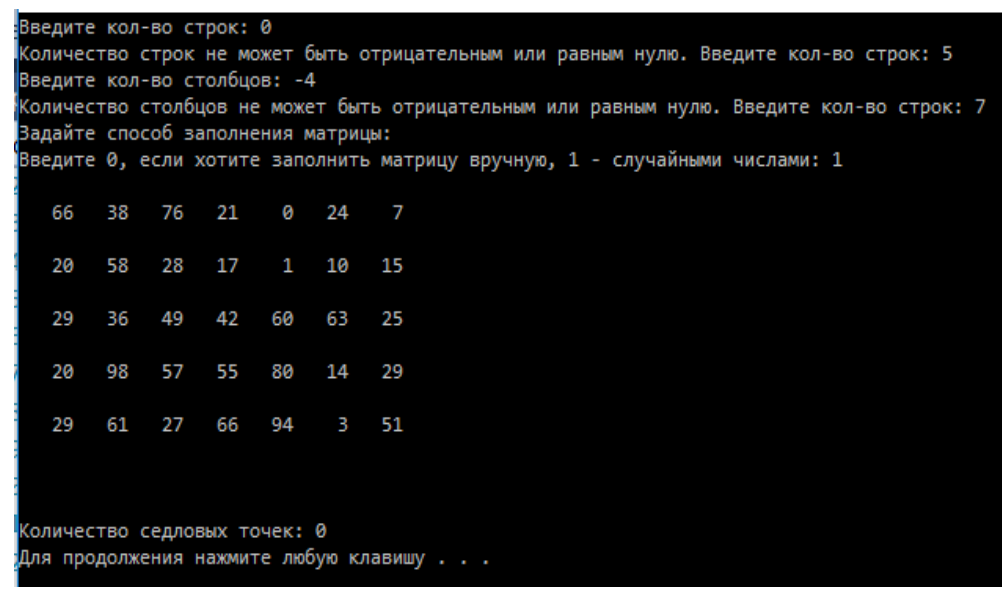

Рис. 9. Проверка на неправильно введенные данные

Все седловые точки совпадают с представленным контрольным примером.

Результат: 1) - 2 седловые точки с индексами $(1 ; 1)$ и $(2 ; 3)$ (рис. 7$)$.

Результат: 2) - 1 седловая точка с индексом $(1 ; 2)$ (рис. 8).

Результат: 3) - проверка на неправильно введенные данные, седловые точки отсутствуют (рис. 9).

\section{Список литературы}

1. Петрова Н.К. Программирование на C++: конспект лекций [Электронный ресурс]. - Режим доступа:https://lms.kgeu.ru/course/view.php?id=2594.

2. Довбуш Г. Visual C++ на примерах / Г. Довбуш, А. Хомоненко. - М.: БХВПетербург, 2012. - 528 с.

3. Балена Ф. Современная практика программирования на MicrosoftVisualBasic и Visual C\# / Ф. Балена, Д. Димауро. - М.: Русская Редакция, 2015. $-640 \mathrm{c}$.

4. Лафоре Р. Объектно-ориентированное программирование в С++ / Р. Лафоре. - 4-е изд. - Питер, 2018. - 928 с.

5. Сидорина Т. Самоучитель MicrosoftVisualStudio C++ и MFC / Т. Сидорина. - М.: БХВ-Петербург, 2014. - 848 с. 


\section{References}

1. Petrova, N. K. Programmirovanie na S++: konspekt lektsii.

2. Dovbush, G., \& Khomonenko, A. (2012). Visual C++ na primerakh. M.: BKhV-Peterburg.

3. Balena, F., \& Dimauro, D. (2015). Sovremennaia praktika programmirovaniia na MicrosoftVisualBasic i Visual C\#. M.: Russkaia Redaktsiia.

4. Lafore, R. (2018). Obektno-orientirovannoe programmirovanie v S++., 928. Piter.

5. Sidorina, T. (2014). Samouchitel' MicrosoftVisualStudio C++ i MFC. M.: BKhV-Peterburg.

Петрова Наталья Константиновна - канд. физ.-мат. наук, доцент кафедры информатики и информационно-управляющих систем, ФГБОУ ВО «Казанский государственный энергетический университет», Россия, Казань.

Petrova Natalya Konstantinovna - candidate of physical and mathemetical sciences, associate professor of the informatics and information management systems department, FSBEI of HE «Kazan State Power Engineering University», Russia, Kazan.

Алимов Тимур Мансурович - студент ФГБОУ ВО «Казанский государственный энергетический университет», Россия, Казань.

Alimov Timur Mansurovich - a student of FSBEI of HE «Kazan State Power Engineering University», Russia, Kazan.

Алабдуллах Мохамад Белал - студент ФГБОУ ВО «Казанский государственный энергетический университет», Россия, Казань.

Alabdullah Mohamad Belal - a student of FSBEI of HE «Kazan State Power Engineering University», Russia, Kazan.

Альхузайи Ахмед Хушам Хусам - студент ФГБОУ ВО «Казанский государственный энергетический университет», Россия, Казань.

Alhuzai Ahmed Husham Hussam - a student of FSBEI of HE «Kazan State Power Engineering University», Russia, Kazan. 
Каттави Раджих Зиад - студент ФГБОУ ВО «Казанский государственный энергетический университет», Россия, Казань.

Kattavi Radzhih Ziad - a student of FSBEI of HE «Kazan State Power Engineering University», Russia, Kazan. 